\title{
Soccer Specific Fitness Differences Across the Common Playing Position Players
}

Received $23^{\text {rd }}$ August 2019 Accepted $16^{\text {th }}$ October 2019 www.ijpefs.com

\author{
Belayneh Chekle ${ }^{a, *}$ and Tefera Tadesse ${ }^{b}$
}

Abstract: As a complex team sport, success in soccer relies on individuals' performance, collective performance, and most importantly on team performance. In soccer, players are usually assigned to a specific position based on the demands of the position and the corresponding qualities of the players. The performance factors are varied and interrelated. As such, whether speed, change-of-direction-speed (CODS) and repeated sprinting ability (RSA) are used to discriminate different position players is not well-established. The purpose of this study was to examine how different position players are different against these parameters. The study participants included 88 soccer players at the Ethiopian National Soccer League classified into six playing positions such as centerbacks, fullbacks, holding-midfielders, attacking-midfielders, outside-midfielders and strikers. The participant players speed, CODS and RSA were measured weekly for about five weeks. The authors analyzed the collected data using one-way ANOVA and Games-Howell multiple comparisons. Results indicated that the position discriminating power of the three measures, including speed, CODS and RSA was found minimal. Among the different position players, only outside-midfielders were significantly higher performers with these qualities measured. Therefore, it was concluded that the performance difference in speed, CODS and RSA among the six position players is not a good discriminator, except for outside-midfielders, who outperformed all other position players (excluding the fullbacks) with the studied parameters.

Key Words: Linear speed, Repeated sprinting ability, Fitness, Playing position

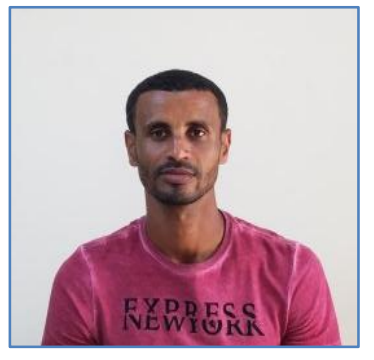

Belayneh Chekle (assistant professor) is a lecturer at Bahir Dar University and is at the verge defending his $P h D$ dissertation at Bahir Dar Unversity. Belayneh Chekle has received his Master's degree in football coaching from Addis Ababa University, Ethiopia. He was following hid PhD study for the last five years and waiting to defend his dissertation at Bahir Dar University, Ethiopia. He is also working as a coach of youth footballers in the sport Academy of Bahirdar University. Since 2017 he is an assistant professor in sport science department. His main research interest is on soccer performance, performance factors, fitness development of junior and elite players and health and exercise

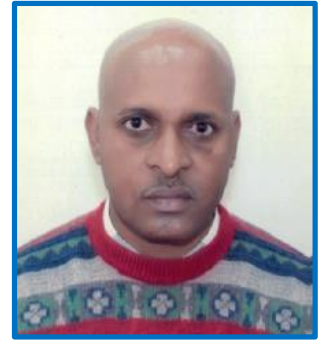

Tefera Tadesse Ph.D. is a Fulbright Postdoctoral Fellow in the Department of Counseling, Educational Psychology, and Special Education at Michigan State University, USA. Dr. Tadesse received his Ph.D. in Curriculum and Instruction from the School of Education in the University of Queensland, Australia. His major research interests are in higher education teaching and learning, student engagement, classroom instruction, cooperative learning, equity and social justice in education, sports sciences, and health sciences. 


\section{Belayneh Chekle and Tefera Tadesse /2019}

\section{Introduction}

Success in contemporary soccer relies on physical fitness to an extent. Surprisingly, the demand of modern soccer drastically changed from the previous decades. This can be witnessed with objective findings that showed the players are performing more explosive movements and competing at higher intensities than ever before $[1$, 2]. Specifically, sprinting is the common action in modern footballers during assisting and scoring situations [3]. Sharing this fact, recent findings even suggest the process of talent identification to critically consider those physical qualities as linear sprinting speed, change-of-direction-speed (CODS) and repeated sprinting ability (RSA) [4]. Moreover, it is shown that there is a noticeable development in sprinting speed in elite male and female soccer players over time [5]. RSA is also found to be one of the most important attribute for higher successful match performance [6]. It has also been argued that games can potentially be won or lost on occasions where repeated sprinting from one or more players and the ability to resist fatigue during these bouts of repeated sprinting is required [7]. This suggests that these qualities are of great relevance to the outcome of the game.

Making the matter more important, it was shown that aerobic capacity is not a distinguishing factor to separate players of different standard [8, 9]. However, anaerobic quality or speed and speed related qualities are crucial factors in soccer. With all the facts, it is possible to claim that improved sprinting skill can make a football player more effective and therefore more valuable. Faster players are probably able to utilize their technical-tactical skill better than their slower counterparts provided that they have identical skills [10]. The chance of dribbling an opponent out of position, or successfully defending an attack, increases with improved sprinting abilities. Thus, soccer players ought to develop these qualities and coaches should take sprinting velocity into account in their professional works. Generally, it is highly acknowledged that teams with higher anaerobic capacities' (sprinting, CODS, lower body strength and RSA) are likely enough to be successful [4]. However, with which position players are these qualities are dominant is not that vivid.

Speed and speed related quality differences are shown to be a function of playing position. For example strikers are significantly faster than defenders [11, 12]. For this, coaches select fastest players for attacking position [13], because they involve in most decisive duels during match play [14]. Still witnessing anaerobic fitness difference among playing position, midfielders are faster than goalkeepers $[10,11]$. The practical evidence of these facts is that when we cater players for a specific position we need to appreciate their physical predisposition. Also during player selection and talent identification evaluating the players with this specific parameter makes the success of coaches, players and teams more likely. During training or conditioning, players in different position should prioritize different physical conditioning regimens to enable them solve position dependent problems during match play. Opposing this, the practical fact indicated that we can have fast and sluggish players in the same position, meaning that the association of speed with playing position is left questionable. My personal and professional observation does not convince me that strikers are the fastest players. We can think of world class strikers and we argue that speed related qualities are not related to only a specific position. We can observe exceptionally speedy players in any position. On the other extreme we can also have sluggish players in different positions (think off Mesut Ozil and Christaino Ronaldo or Mabpe in the wing, Olivier Giroud, Manzugic or Romelu Lukaku in the striker and Gorginho, Matic, Kante and Sisoko in holding-mid). Thus the relationship of position and speed or speed related quality in today`s soccer is not that clear.

RSA during match is also believed to be position specific in soccer players. He also noticed that outside-midfielders significantly performed more sprints (RSA) than all other position players during match play. It was also found that strikers perform more sprint than fullbacks, fullbacks more than midfielders and midfielders perform more sprint than center-backs [15]. Still he found that holding-midfielders perform more sprints than 


\section{Belayneh Chekle and Tefera Tadesse /2019}

attacking-midfielders. Generally, the issue of which position players are faster in linear sprinting speed and CODS is not clearly known. Still which position players are better in performing consecutive sprints with only a brief recovery between the sprints needs to be elucidated. Therefore, the prime purpose of this study was to disclose which position players are faster in a soccer specific field test of linear sprinting and changing-of-direction-speed. It was also aimed to unveil which position players are capable of producing the best possible sprint over consecutive sprints. This could potentially help to be informed practitioners on training and to help clarify the need of having functional or individual conditioning. It can also have an immediate significance of informing how fit are the studied players to the demand of the position that they are assigned.

\section{Method}

A total of 88 competitive soccer players from the third level of league in the nation participated in the study for about five weeks. Using selected soccer specific fitness parameters, different position players were compared. Speed, CODS and RSA performance of the players were measured weekly for about five weeks period. Each player was tested five times throughout the study. For sprinting speed 40-m dash and for CODS 9-3-6-3-9 test protocol was used. The test procedure involves the same kind of warming up routine and each of the players given 3 trails with 710 minutes recovery between trials and the best time was taken. For RSA, 6*35-m test with 25 seconds recovery between consecutive sprints was used. For each fitness, the participants were measured/observed 5 times (a total of 440 observations were made). One-way ANOVA with Games-Howell post-hoc test was used for analysis

\section{Result}

A total of 440 observations were made for linear sprinting speed over $40 \mathrm{~m}$ dash, CODS in 9-3-63-9 test and RSA with $6 * 35 \mathrm{~m}$ test protocol. Here under is the mean time score or performance of the different position players (table 1).

\subsection{Sprinting Speed}

Linear sprinting speed over $40 \mathrm{~m}$ was assessed to examine how different are the players based on their playing position. The players categorized in to six different positions as centerback, fullback, holding-midfielders, attackingmidfielders, oust-side-midfielders (wingers) and strikers. For this, a total of 88 players tested weekly for about five consecutive weeks. Thus, 440 observations were made for analysis.

The different position players were significantly different in their sprinting speed performance $F(5,434)=11.60, p<.001$ (table 2). The post hoc result, Games-Howell, revealed that centerbacks were significantly faster than holdingmidfielders and slower than outside-midfielders $(\mathrm{p}=.001)$. However, center-backs were not different from full-backs, attacking-midfielders and strikers. Fullbacks do not differ significantly from any position players. Holding-midfielders were significantly sluggish than center-backs $(p=.011)$, wingers $(p<.001)$ and strikers $(p=.024)$. Holding-midfielders do not have a significant difference from fullbacks and attacking-midfielders. Attacking-midfielders were significantly slower than out-side-midfielders $(p<.001)$. But they do not significantly differ from other position players. However, outside-midfielders were significantly faster than center-backs $(p=.001)$, holding-midfielders $(\mathrm{p}<.001)$, attacking-midfielders $(p<.001)$ and strikers $(p<.001)$. But they do not outperform that of fullbacks. Though they do have a lower mean time, they were not significantly faster than fullbacks. The strikers were faster than holdingmidfielders $(\mathrm{p}=.024)$. However in sprinting speed, they were significantly outperformed by outsidemidfielders $(p<.001)$. Still they do not differ significantly from other position players. Eta-squared ( 12 ) as it can be calculated from the figures of between group sum of squares and total is 0.118 (11.8\%).

\subsection{CODS}

For a time period of about five weeks, the participants were tested weekly with the 9-3-6-3-9 CODS test protocol. The test was specifically designed to assess how fast the players were in changing their 


\section{Belayneh Chekle and Tefera Tadesse /2019}

direction and the required distance (24-m forward and 6-m backward). To examine how the different position players differs with this parameter, one-way ANOVA was used with appropriate post-hoc-test, Games-Howell.

Table 1 Descriptive Statistics of the players' fitness performance in seconds

\begin{tabular}{|c|c|c|c|c|c|c|c|}
\hline \multirow[t]{2}{*}{ Position } & \multirow[t]{2}{*}{$\mathrm{N}$} & \multirow{2}{*}{$\begin{array}{l}\text { Speed } \\
\text { (Seconds) }\end{array}$} & \multirow{2}{*}{$\begin{array}{l}\text { CODS } \\
\text { (seconds) }\end{array}$} & \multicolumn{4}{|c|}{ RSA (seconds) } \\
\hline & & & & RSA total & $\begin{array}{l}\text { RSA } \\
\text { average }\end{array}$ & $\begin{array}{l}\text { RSA } \\
\text { best }\end{array}$ & $\begin{array}{l}\text { RSA } \\
\text { worst }\end{array}$ \\
\hline Center Backs & 110 & 5.6888 & 9.8973 & 31.2735 & 5.2187 & 5.0335 & 5.3561 \\
\hline Fullbacks & 30 & 5.5990 & 9.9023 & 30.7593 & 5.1266 & 5.0197 & 5.2373 \\
\hline Holding midfielders & 70 & 5.8420 & 10.4137 & 31.8267 & 5.3045 & 5.1546 & 5.4353 \\
\hline Attacking midfielders & 90 & 5.7679 & 10.2759 & 31.3458 & 5.2243 & 5.0830 & 5.3629 \\
\hline Outside midfielders & 85 & 5.4418 & 9.2528 & 29.5710 & 4.9285 & 4.7859 & 5.0767 \\
\hline Strikers & 55 & 5.7002 & 9.9996 & 31.1400 & 5.1900 & 5.0602 & 5.3356 \\
\hline
\end{tabular}

Table 2 Linear sprinting speed among different position players

\begin{tabular}{lccccc}
\hline & Sum of Squares & df & Mean Square & F & Sig. \\
\hline Between Groups & 7.580 & 5 & 1.516 & 11.600 & .000 \\
Within Groups & 56.721 & 434 & .131 & & \\
Total & 64.301 & 439 & & & \\
\hline
\end{tabular}

Table 3 CODS performance among different position players

\begin{tabular}{lrrrrr}
\hline & Sum of Squares & \multicolumn{1}{c}{ df } & Mean Square & F & Sig. \\
\hline Between & 66.426 & 5 & 13.285 & 11.589 & .000 \\
Groups & & & & & \\
Within Groups & 497.521 & 434 & 1.146 & & \\
Total & 563.947 & 439 & & & \\
\hline
\end{tabular}

Table 4 RSA across the different position players

\begin{tabular}{llrrrrr}
\hline & & Sum of Squares & df & Mean Square & \multicolumn{1}{c}{ F } & \multicolumn{1}{c}{ Sig. } \\
\hline \multirow{3}{*}{ RSA Total Time } & Between Groups & 243.220 & 5 & 48.644 & 20.125 & .000 \\
& Within Groups & 1049.028 & 434 & 2.417 & & \\
& Total & 1292.248 & 439 & & & \\
RSA Average & Between Groups & 6.825 & 5 & 1.365 & 20.332 & .000 \\
& Within Groups & 29.138 & 434 & .067 & & \\
& Total & 35.964 & 439 & & & \\
RSA Best & Between Groups & 6.390 & 5 & 1.278 & 13.258 & .000 \\
& Within Groups & 41.833 & 434 & .096 & & \\
& Total & 48.223 & 439 & & & \\
RSA Worst & Between Groups & 6.395 & 5 & 1.279 & 18.727 & .000 \\
& Within Groups & 29.639 & 434 & .068 & & \\
& Total & 36.033 & 439 & & & \\
\hline
\end{tabular}

The players from the six position differ significantly in their RSA total time, $F(5,434)=20.12$, $\mathrm{p}<.001$, RSA mean time $\mathrm{F}(5,434)=20.33, \mathrm{p}<.001$, RSA best time $\mathrm{F}(5,434)=13.26, \mathrm{p}<.001$ and RSA worst time $F(5,434)=18.72, \mathrm{p}<.001$ (table 4$)$ 


\section{Belayneh Chekle and Tefera Tadesse /2019}

The players in the different position differ significantly in their performance or test-score of 93-6-3-9 CODS F $(5,434)=11.59, \mathrm{p}<.001$. The GamesHowell multiple comparison revealed that centerbacks did not differ in their performance from fullbacks, attacking-midfielders, and strikers. However, center-backs were significantly faster than holding-midfielders $(p=.005)$ and slower than wingers $(\mathrm{p}=.008)$. Fullbacks did not have a significant difference from any position players in CODS.

Holding-midfielders were significantly slower (sluggish) than center-backs $(p=.005)$, outsidemidfielders $(p<.001)$ and strikers $(p=.023)$. Holdingmidfielders do not differ from fullbacks and attacking-midfielders to a significant level. Attackingmidfielders were significantly slower than outsidemidfielders $(\mathrm{p}<.001)$, though they do not differ from any other position players in changing their direction. The fastest players, outside-midfielders, significantly outperform center-backs $(p=.008)$, holding-midfielders $(\mathrm{p}<.001)$, attacking-midfielders $(p<.001)$ and strikers $(p<.001)$. However, wingers do not outperform fullbacks to a significant level. The strikers, who are expected to be the fastest in changing direction, were able to be faster than only holding-midfielders $(p<.023)$. They do not have that significant difference from any other position players. However the effect size (Eta-squared ( $\eta 2$ )) which can be calculated using between groups sum of squares and total sum of squares was small ( $\eta 2=0.118)$, indicating that CODS position discriminating power is not that big.

\subsection{RSA}

RSA performance of the players over $6 * 35-\mathrm{m}$ was considered using total time, average time, best time and worst time. Using these parameters, the level of performance in different position players was examined using one-way ANOVA.

In terms of RSA total time, center-backs were significantly outperformed by outside-midfielders $(p<.001)$. But they do not have a significant performance difference from other position players in this regard. These way fullbacks do not differ from any position players. However, holding-midfielders significantly outperformed by outside-midfielders $(p<.001)$ and strikers $(p=.004)$. However, holdingmidfielders do not have that significant difference from other position players. Still attackingmidfielders do not differ from any position players, except wingers, who outperform them $(p<.001)$. The outside-midfielders are the one to outperform all position players $(\mathrm{p}<.001)$, except the fullbacks. There is no a statistically significant difference between outside-midfielders and fullbacks, though the mean time score of outside-midfielders was better. Strikers outperform only holding-midfielders $(\mathrm{p}=.004)$ and outperformed by outside-midfielders $(\mathrm{p}<.001)$ and wingers.

RSA mean time was different the same way RSA total time does. Outside-midfielders outperform center-backs $\quad(\mathrm{p}<.001), \quad$ attacking-midfielders $(p<.001)$ and strikers $(p<.001)$. Their performance in this regard was not significantly superior to fullbacks however. Holding-midfielders outperformed by also strikers $(\mathrm{p}=.004)$. Holding-midfielders do not got significantly outperformed by other position player, though they are the slower players with their mean time measure.

Against RSA best time, center-backs do not differ from fullbacks, holding-midfielders, attackingmidfielders and strikers. They were only significantly slower than outside-midfielders $(\mathrm{p}<.001)$. Fullbacks do not have a significant difference from all other position players. Holding-midfielders significantly outperformed by outside-midfielders $(\mathrm{p}<.001)$. But their difference was not significant when compared with other position players. Outside-midfielders outperform all other positions $(\mathrm{p}<.001)$ and at a significance level of $\mathrm{p}=.012$ with fullbacks.

When players compared based on RSA worst time, center-backs do not have that significant difference with all positions, except outsidemidfielders $(p<.001)$ (appendix). Still fullbacks do not have a significant difference with all position players in this regard. The same way, holdingmidfielders do not have that significant difference with center-backs, attacking-midfielders, centerbacks, or strikers. They were significantly inferior to wingers only $(p<.001)$. Attacking-midfielders were significantly slower than outside-midfielders 


\section{Belayneh Chekle and Tefera Tadesse /2019}

( $\mathrm{p}<.001)$. Outside-midfielders actually achieved the smaller worst time when they are compared with center-backs, holding-midfielders, attackingmidfielders and strikers $(\mathrm{p}<.001)$. In this regard, outside-midfielders were not significantly superior to fullbacks. Strikers were also significantly inferior to wingers $(p<.001)$, though were not significantly different from other position players. Generally, regardless of the significant difference among different position players, the effect size ( $\eta 2$ ) was $0.188,0.190,0.133$ and 0.177 for RSA total time, average time, best time and worst time respectively, which are small.

\section{Discussion}

The players from wing, striker, and fullbacks' were expected to be the fastest in sprinting speed and related factors [10, 11, 13]. Still when we consider world class outside-midfielders and fullbacks they are among the fastest players and the finding of this with regard to wingers goes in parallel with this. The role fullbacks have in their specific position involves a lot of sprinting when defending and as well attacking. Wingers, the fastest footballer can otherwise outrun and create spaces easily. They are expected to be faster than other position players. However, the finding in this study do noes goes in parallel with this fact and logic, meaning that strikers and fullbacks were not that significantly different from other position players. We can question that, we are not good in assigning position or in developing position specific qualities. Our football status can be accounted to this gap to an extent. Because, fullbacks are players who involve equally in the attacking and defensive situations and importantly they face wingers, the fastest player, so that they are deemed to be speedy. And when players involve in attacking situation (as assisting and scoring player) they are inherently expected to sprint and create space [3].

Inherent to the nature or demand of the position, fullbacks and strikers were expected to outperform most position players in changing-ofdirection-speed. However the finding in this study does not align with this assumption. Specific to the context, those players we assign in this position were not in accordance with their quality. And the other reason may be the trend and quality of individual conditioning that we use may be problematic. The test protocol used can also have its limitation in mimicking the typical movement pattern of fullbacks for which they are too accustomed. In this regard test protocols need to be critically examined in their ability to be too specific enough in addressing the specific and typical movement pattern or natures. The same way with this study, Fiorilli et al. (2017), found no significant difference in CODS performance among different position players [16]. However, one weakness with this study was that players were only classified as defenders, midfielders and forwards. This classification of players lacks consideration of the different demand of each position and players. For example being defender, fullbacks and centerbacks are assumed to be different in terms of fitness and other technical-tactical issues.

Generally, each fitness test should consider each position demand or typical movement pattern. For example, the kind of change-of-direction and sprint that fullbacks typically perform may be different from holding-midfielders, center-backs, strikers or attacking-midfielders. Thus, a specific kind of test protocol may be necessary for each position. Speed test for strikers need to be different from the speed test protocol of center-backs, holdingmidfielders or fullbacks. Detailed movement pattern analysis for each position should be done to devise a relevant fitness test protocol for each position. For instance, 9-3-6-3-9 CODS test protocol was found too relevant for strikers [12]. Therefore, for fullbacks to be similar with other position players it can be accounted to the weakness of the CODS (9-3-6-3-9) test protocol to lack specificity, or criterion validity for the position movement pattern (quality needed). Still fullbacks were not significantly outperformed by wingers (the fastest).

Mostly strikers are expected to be the faster than most position players to gain an advantage in deceiving and taking-over their opponents. But with this finding, they were not found that faster. In today`s soccer however, some of the world class strikers are not that speed than other position players (for example, Olivier Giroud of Chelsea, Harry Kane of Spurs, Firmino of Liverpool, Benzema of Real 


\section{Belayneh Chekle and Tefera Tadesse /2019}

Madrid et.,). Other physical qualities and technicaltactical ability can compensate this. Thus, the result of this finding with CODS performance of the strikers is too persuading.

One of the visible finding in this study was that those players who were good in linear sprinting speed were found to be better in the RSA or CODS performance. Other findings on the matter also showed that sprinting speed is a great influencing factor for RSA best and RSA mean [17, 18]. LopezSegovia et al. (2015) assured that there is statistically significant relationship between muscular strength and the first three sprints of RSA test ( $r=-.064$, $\mathrm{p}<.005$ ) [17]. Thus, it can be claimed that sprinting speed is a significant predictor of overall RSA performance [18].

Low-volume high-intensity interval training (HIT) appears to be an efficient and practical way to develop RSA [19], but additional speed endurance training is associated with an improved ability to perform repeated high-intensity work [20] because RSA also correlates with $\mathrm{VO}_{2} \max$ [21]. Thus, the smaller performance difference of different position players of the studied teams can be accounted to the lack of individual or position specific functional trainings. The training that we aim for speed or RSA should be in a close adherence to the characteristics of speed and RSA and principles of specificity (position demand) and individual difference. For this a number of performance tests have been developed in order to evaluate RSA in soccer players with the aim of identifying potential weaknesses and prescribe individualized training protocols tailored specifically to the demands of different positional roles [22]. Typically these tests incorporate sprint distances of between 15 and 40-m with 3-15 repetitions, while recovery periods last for 15-30 seconds [5].

\section{Conclusion}

The six position players were significantly different in their performance in sprinting speed, CODS and RSA measures. These anaerobic fitness parameters were found position specific. However the strikers were not the fastest players. Instead the outside-midfielders were the best performers or conditioned with these soccer specific fitness parameters of linear sprinting speed, change-ofdirection-speed and repeated sprinting ability. Still, fullbacks like that of strikers were not dominant with these fitness qualities. The effect size or the differences however were not that big enough to discriminate each position.

The other visible result with the study was that, players who were good in linear sprinting speed were found to perform better in CODS and in all RSA measures.

Further researches on the matter should focus on how significant is the performance level of different position players with a due consideration of how the participant players are having position specific functional training, hoe the tactical roles assigned by the coaches matter the player physical taxation and how long the players were accustomed and played in that specific position. Furthermore, the typical playing style of teams of the participating players and the favourite defensive and attacking mentality of the players need to be highly considered as all can impact the stress which can be imposed the player and the subsequent adaptation.

\section{References}

[1] P.S. Bradley, W. Sheldon, B. Wooster, P. Olsen, P. Boanas, \& P. Krustrup, High-intensity running in English FA Premier League soccer matches, Journal of Sports Sciences, 27 (2009) 159-168.

[2] J.F. Da Silva, L.G.A. Guglielmo, \& D. Bishop, Relationship Between Different Measures of Aerobic Fitness and Repeated-Sprint Ability in Elite Soccer Players, Journal of Strength and Conditioning Research, 24 (2010) 2115-2121.

[3] O. Faude, T. Koch, \& T. Meyer, traight sprinting is the most frequent action in goal situations in professional football, Journal of Sports Sciences, 30 (2012) 625-631.

[4] K.D. Dodd, \& T.J. Newans, Talent identification for soccer: Physiological aspects, Journal of Science and Medicine in Sport, 21 (2018) 10731078. 


\section{Belayneh Chekle and Tefera Tadesse /2019}

[5] T. Haugen, E. Tonnessen, J. Hisdal, \& S. Seiler, The role and development of sprinting speed in soccer. Brief review, International Journal of Sports Physiology and Performance, 9 (2014) 432-441.

[6] H.Å. Andersson, M.B. Randers, A. Heiner-Møller, P. Krustrup, \& M. Mohr, Elite Female Soccer Players Perform More High-Intensity Running When Playing in International Games Compared With Domestic League Games, Journal of Strength and Conditioning Research, 24 (2010) 912-919.

[7] O. Girard, A. Mendez-Villanueva, \& D. Bishop, Repeated-Sprint Ability - Part I. Sports Medicine, 41 (2011) 673-694.

[8] P.S. Bradley, C. Carling, A. Gomez Diaz, P. Hood, C. Barnes, J. Ade, M. Mohr, Match performance and physical capacity of players in the top three competitive standards of English professional soccer, Human Movement Science, 32 (2013a) 808-821.

[9] E. Tonnessen, E. Hem, S. Leirstein, T. Haugen, \& S. Seiler, Maximal aerobic power characteristics of male professional soccer players, 1989-2012, International Journal of Sports Physiology and Performance, 8 (2013) 323-329.

[10] C. Carling, F. Le Gall, \& G. Dupont, Analysis of repeated high-intensity running performance in professional soccer, Journal of Sports Sciences, 30 (2012) 325-336.

[11] J. Boone, R. Vaeyens, A. Steyaert, L.V. Bossche, \& Bourgois, J. Physical Fitness of Elite Belgian Soccer Players by Player Position, Journal of Strength and Conditioning Research, 26 (2012) 2051-2057.

[12] G. Sporis, I. Jukic, L. Milanovic, \& V. Vucetic, Reliability and Factorial Validity of Agility Tests for Soccer Players, Journal of Strength and Conditioning Research, 24 (2010) 679-686.

[13] M. Buchheit, A. Mendez-villanueva, B.M. Simpson, \& P.C. Bourdon, Repeated-Sprint Sequences During Youth Soccer Matches, International Journal of Sports Medicine, 31 (2010) 709-716.

[14] E. Rampinini, A. Coutts, C. Castagna, R. Sassi, \& F. Impellizzeri, Variation in Top Level Soccer
Match Performance, International Journal of Sports Medicine, 28 (2007) 1018-1024.

[15] J. Schimpchen, S. Skorski, S. Nopp, \& T. Meyer, Are "classical" tests of repeated-sprint ability in football externally valid? A new approach to determine in-game sprinting behaviour in elite football players, Journal of Sports Sciences, 34 (2015) 519-526.

[16] G. Fiorilli, M. Mitrotasios, E. Iuliano, E.M. Pistone, G. Aquino, G. Calcagno, \& A. Di Cagno, Agility and change of direction in soccer: differences according to the player age, The Journal of Sports Medicine and Physical Fitness, 57 (2017) 1597-1604.

[17] M. López-Segovia, F. Pareja-Blanco, P. JiménezReyes, \& J. González-Badillo, Determinant Factors of Repeat Sprint Sequences in Young Soccer Players, International Journal of Sports Medicine, 36 (2014), 130-136.

[18] W. Dardouri, M.A. Selmi, R.H. Sassi Z. Gharbi, A. Rebhi, M-H. Yahmed, \& W. Moalla, Relationship between Repeated Sprint Performance and both Aerobic and Anaerobic Fitness, Journal of Human Kinetics, 40 (2014) 139-148.

[19] L. Cipryan, G. Tschakert, \& P. Hofmann, Acute and post-exercise response to high-intensity interval training in endurance and sprint athletes, Journal of Sports and Medicine, 16 (2017) 219-229.

[20] M. Nyberg, M. Fiorenza, A. Lund, M. Christensen, T. Rømer, P. Piil, J. Bangsbo, Adaptations to Speed Endurance Training in Highly Trained Soccer Players, Medicine \& Science in Sports \& Exercise, 48 (2016) 13551364.

[21] V. Di Salvo, W. Gregson, G. Atkinson, P. Tordoff, \& B. Drust, Analysis of High Intensity Activity in Premier League Soccer, International Journal of Sports Medicine, 30 (2009) 205-212.

[22] C. Barnes, D. Archer, B. Hogg, M. Bush, \& P. Bradley, The Evolution of Physical and Technical Performance Parameters in the English Premier League. International Journal of Sports Medicine, 35 (2014) 1095-1100. 


\section{Funding}

This study was not funded by any grant

\section{Acknowledgements}

The authors would like to thank every participant for his effort and time.

\section{Conflict of interest}

None of the authors have any conflicts of interest to declare.

\section{Informed consent}

All participants gave written informed consent to participate in this study.

\section{About The License}

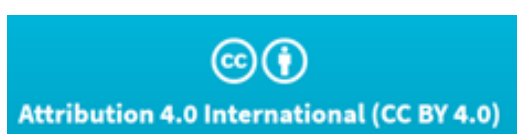

The text of this article is licensed under a Creative Commons Attribution 4.0 International License 